\title{
Extending indications for minimally invasive esophagectomy to pediatric patients: The same operation...just smaller?
}

\author{
Inderpal S. Sarkaria, MD, FACS
}

\footnotetext{
From the Department of Cardiothoracic Surgery, University of Pittsburgh School of Medicine and University of Pittsburgh Medical Center, Pittsburgh, Pa.

Disclosures: Author has nothing to disclose with regard to commercial support.

Received for publication July 11, 2016; accepted for publication July 12, 2016; available ahead of print Sept 1, 2016.

Address for reprints: Inderpal S. Sarkaria, MD, FACS, Department of Cardiothoracic Surgery, University of Pittsburgh Medical Center, UPMC Presbyterian-Shadyside, 5200 Centre Ave, Suite 715.27, Pittsburgh, PA 15232 (E-mail: sarkariais@upmc.edu)

J Thorac Cardiovasc Surg 2016;152:e117

$0022-5223 / \$ 36.00$

Copyright (c) 2016 by The American Association for Thoracic Surgery

http://dx.doi.org/10.1016/j.jtcvs.2016.07.040
}

The use of minimally invasive esophagectomy (MIE) has gained considerable traction in adult patients requiring resection for neoplastic or benign conditions.

In the current case, Majors and colleagues ${ }^{1}$ report a case of complete laparoscopic and thoracoscopic MIE in a 6-year-old girl with long-segment stricture after caustic injection. It is encouraging to see a potential extension of indications to an operation increasingly shown to carry decreased morbidity while maintaining outcomes equivalent to open procedures. However, there are several important caveats that cannot be understated in this case.

First and foremost, although this single-case report demonstrates the feasibility of MIE in pediatric patients, care must be taken not to extrapolate the outcomes broadly, especially in terms of safety. Performance of MIE requires a demanding skill set acquired over a significant learning curve. It is arguable these skills are best attained through a dedicated period of advanced specialty training at highvolume centers experienced in these operations (as is the case with the article's senior author [B.W.]). In other words, this is not routine surgery, and careful consideration must be given to the manner in which these operations may be approached in the relatively rare circumstances in which esophagectomy may be required in the pediatric population. Likely, partnerships between surgeons with individual expertise in MIE and pediatric operations may be envisioned to ensure best outcomes and minimize technically avoidable morbidity.

Second, although many of us bear an inherent bias toward assumed added value with minimally invasive surgical approaches (this author included), it is admittedly difficult to extrapolate the benefit of MIE in terms of morbidity given the significant differences between the adult and pediatric populations in terms of comorbid conditions, presence of cancer, consequences of adjuvant therapies such as radiation and chemotherapy, obesity, and overall physical

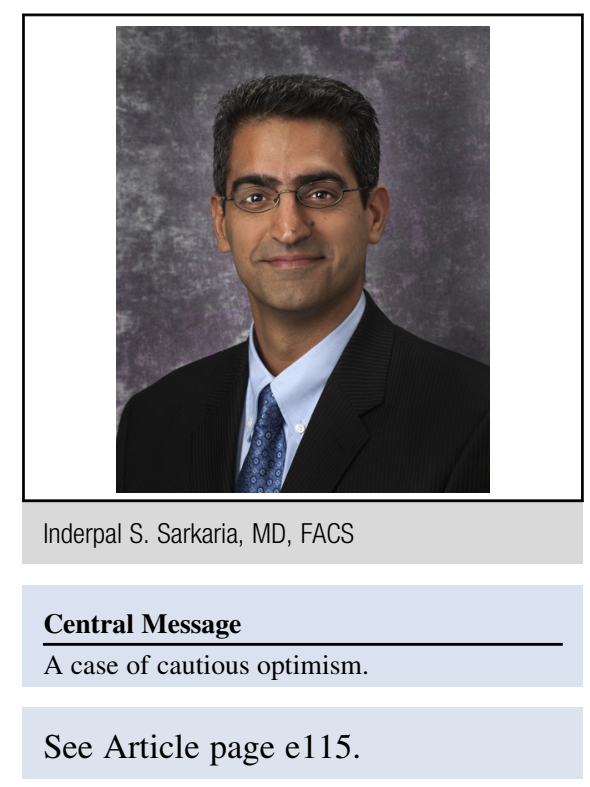

reserve. This, again, must be gauged within the perspective of relative risk versus relative benefit.

Finally, the authors outline several relevant differences in the conduct of anesthesia and technical aspects of the operation distinct from the "standard" MIE as performed by the senior author in the adult population. It is likely the "key" components of MIE that may confer benefit in the pediatric population remain to be determined. Is it just a smaller MIE, with the same extrapolated impact on outcomes? As per the authors' own reasoning in opting for a minilaparotomy, could it be argued that the majority of the abdominal operation may have been performed through the self-same incision with less time and with similar physical impact to the patient?

Caveats and broad-scope critiques aside, the reported case appropriately functions as an encouraging demonstration of feasibility in the pediatric population and potential "proof of principle" to consider extended indications for MIE in these patients, albeit with an ever tempered and keen eye toward maintaining safety and optimal outcomes.

\section{Reference}

1. Majors J, Zhuge Y, Eubanks T, Weksler B. Minimally invasive esophagectomy in a 6-year-old girl for the sequelae of corrosive esophagitis. J Thorac Cardiovasc Surg. 2016;152:e115-6. 